\title{
Things molecules can do
}

\author{
Artificial molecular machines are scientifically satisfying structures.
}

S tudying molecules is a fascinating intellectual endeavour. Within a molecule, each atom is in exactly the right position to accomplish something useful. There is beauty in appreciating such a sense of purpose and chemists have developed a soft spot for beautiful molecules, recognizing their simplicity, elegance and precise atomic arrangements. Whereas the instinct of the organic chemist may be to find the most economical path to make a molecular structure, the instinct of the physical chemist is to see what a molecule can do.

There are molecules walking on tracks, molecules that can toggle between configurations and properties, molecules that can do work on the environment, molecules that can catalyse their own synthesis, molecules that can propel themselves in solution and even molecules that can assemble building blocks to make another molecule. In many cases, these examples are inspired by natural molecules, such as myosin, ATP and rhodopsin. Being able to reproduce the behaviours of these natural molecules, even in a minimalistic form (any of these synthetic molecules pale in comparison to the sophistication of biomolecules), teaches us something about natural processes, for making is understanding.
The latest addition to this conceptual journey in the molecular world can be found in this issue in a Letter by De Bo et al. ${ }^{1}$. The authors have made a molecule that can synthesize a second molecule, which in turn assumes a characteristic conformation that can catalyse the formation of a third molecule. In other words, the work shows a molecule that can synthesize a chemically functional molecule. This is the closest we have been to being able to mimic the behaviour of a ribosome.

The initial molecule is a rotaxane, the axle of which is a polymer functionalized with leucine esters. The ring of the rotaxane travels the polymeric backbone, picking up amino acid residues to form an oligopeptide. This oligomer, together with the ring to which it is attached, slips off from the axle and folds itself into an alpha-helix, which finally catalyses the formation of an asymmetric epoxide from a chalcone in solution. The similarities with a ribosome are obvious: the molecule transforms the information contained in the polymer backbone into the secondary structure of a new molecule, which then does something chemically useful.

Some could see the resemblance of this approach with a previous system in which a similar rotaxane could pick up three amino acids and make a tripeptide before slipping it off the axle $e^{2}$. The main novelty here is the incorporation of the polymer backbone. The formation of the epoxide (via the Julià-Colonna reaction) can be carried out using much cheaper compounds than a rotaxane. Plus, the ring takes an average of $96 \mathrm{~h}$ to move along the backbone, not exactly a speedy process!

It is important to understand, we believe, that the point of making molecular machines is not - as of yet - to make practically useful structures. As has been said: "there is as yet no task that can be performed by a synthetic molecular machine that cannot be done more effectively another

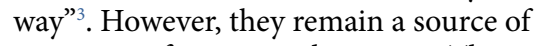
inspiration for nanoscale science. The simple fact that we can take an amazingly complicated natural process, strip it down to its essential components and build an artificial system that can reproduce its behaviour is scientifically satisfying. And there is beauty in that.

Published online: 4 May 2018

https://doi.org/10.1038/s41565-018-0155-6

References

1. De Bo, G. et al. Nat. Nanotech. https://doi.org/10.1038/s41565018-0105-3 (2018).

2. Lewandowski, B. et al. Science 339, 189-193 (2013).

3. Leigh, D. A. Angew. Chem. Int. Ed. Engl. 55, 14506-14508 (2016).

\section{Genius in science, genius in teaching}

\author{
We celebrate Feynman's work as scientist and communicator on the one hundredth anniversary of his birth.
}

$\mathrm{R}$ chard Phillips Feynman is arguably one of the most well-known scientists of the twentieth century. As a

theoretical physicist, he contributed to the development of quantum electrodynamics, most notably through the conception of a method to represent the interaction between fundamental particles via simple graphical representations, now known as Feynman diagrams. He also contributed to high energy physics, and he is known to have been part of the Manhattan Project and of the committee investigating the 1986 Space Shuttle Challenger disaster.

Feynman's contributions to science, which earned him the Noble Prize in Physics in 1965, are undisputed. Still, what really makes him special among other eminent scientists is his role as a communicator. Many of us know him through the books that he wrote or co-wrote, the autobiographical Surely You're Joking, Mr Feynman! and Why Do You Care What Other People Think?, and the more scientific Feynman Lectures on Physics and Six Easy Pieces, among others. Through his love of science and his sense of humour he was able to make difficult concepts more approachable. Often, he achieved this through a non-canonical approach such as in his 'fourth easy piece' about the conservation of energy law, to cite just one example. Perhaps his most valuable lesson was that the beauty of nature can be seen through the eyes of a scientist, and not just through those of an artist or a poet.

Feynman would have been 100 years old this month. He has often been celebrated as the inspirer of nanotechnology with his 'plenty of room' lesson. This is not why we remember him here. Feynman was passionate about physics and science more generally, but also about music and art. He loved life in all its aspects. This is what made him such an inspiring figure, and for this we wish to celebrate his work and his life. Happy Birthday, Mr Feynman!

Published online: 4 May 2018

https://doi.org/10.1038/s41565-018-0148-5 